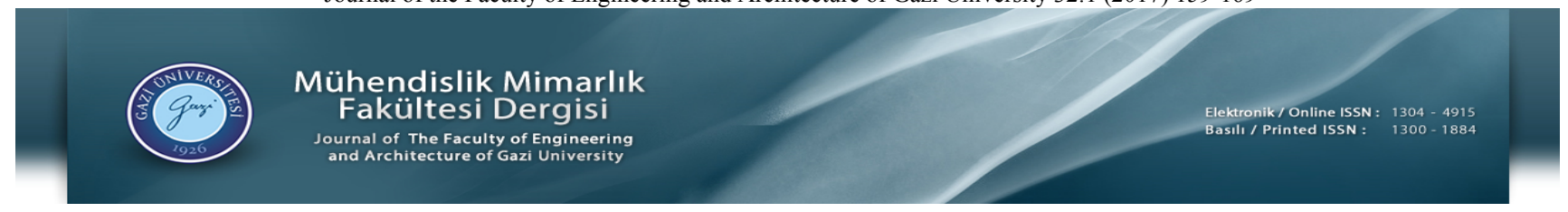

\title{
Radyal havalı yataklarda basınç dağılımının nümerik analizinde çözüm parametrelerinin ve silindirik grid birleşiminin etkileri
}

Abdurrahim Dal*, Tuncay Karaçay

Gazi Üniversitesi, Mühendislik Fakültesi, Makine Mühendisliği Bölümü, Ankara, Türkiye

\section{Ö N E Ç I K A N L A R}

- Daha büyük boşluk değerlerine sahip basınçlı hava yatakları için (c=250 $\mu$ m'ye kadar) basınç dağılımlarının hesaplanması

- $\quad$ Reynold's denkleminin Değişen Yönlü Nümerik İntegrasyon Metodu ile çözümü

- Çözüm gridi birleşme çizgisindeki basınç değerlerinin iteratif olarak hesaplanması için yeni bir yöntem

Makale Bilgileri

Geliș: 24.12.2015

Kabul: 29.03 .2016

DOI:

10.17341/gazimmfd.300607

Anahtar Kelimeler:

Basınçlı hava yatakları, reynold's denklemi,

değişen yönlü kapalı

nümerik integrasyon metodu

\section{ÖZET}

Basınçlı hava yatakları ile desteklenmiş yatak-rotor sisteminde, yatak ve rotor arasındaki havanın akıșı yatak-rotor sisteminin dinamiğini doğrudan etkilemektedir. Bu akışın modellenmesi ve modelin çözümü basınçlı hava yatakları için oldukça önemlidir. Havanın akışı Reynold's denklemi ile modellenmekte ve nümerik çözüm metotları kullanılarak yatak ve rotor arasındaki basınç dağılımları hesaplanmaktadır. Basınç dağılımının elde edilmesi için kullanılan yöntemlerden bir tanesi de Değișen Yönlü Kapalı Nümerik İntegrasyon yöntemidir. Ancak bu metotta özellikle yatak ve rotor arasındaki boşluk miktarının artması $(\mathrm{c}>100 \mu \mathrm{m})$ ile birlikte ıraksama problemleri ortaya çıkmaktadır. Bu çalışmada, büyük boşluk değerlerine sahip basınçlı hava yatakları için ( $\mathrm{c}=250 \mu \mathrm{m}$ 'ye kadar) zaman adımı, grid boyutları ve yakınsama kriteri gibi nümerik çözüm parametrelerinin ve silindirik grid birleşiminin yatak ve rotor arasındaki basınç dağılımına etkileri araştırılmıștır.

\section{Effects of numerical solution parameters and boundary condition of grid on pressure distribution of radial air bearing}

\section{H I G H L I G H T S}

- Stable calculation of pressure distribution for externally pressuresized air bearing with high values of radial clearance (up to $\mathrm{c}=250$ $\mu \mathrm{m})$

- Numerical solution of Reynold's equation with using Alternating direction implicit method

- A new method for iterative calculation of pressure values on the boundary integration of the numerical solution grid

Article Info

Received: 24.12 .2015

Accepted: 29.03 .2016

DOI:

10.17341/gazimmfd.300607

Keywords:

Air bearing, reynold's equation, alternating direction implicit method

\section{ABSTRACT}

In the externally pressurized air bearing-rotor system, characteristic of air flow between rotor and bearing directly effects dynamics of bearing-rotor system. Mathematical model of the air flow and numerical solution of this model are very important in externally pressurized air bearing. The air flow is modelled using Reynold's equation and pressure distribution is obtained using numerical solution methods. One of these numerical solution methods is Alternating Direction Implicit method used to obtain pressure distribution between rotor and bearing. However, this method has convergence problems especially with increasing radial clearance $(\mathrm{c}>100 \mu \mathrm{m})$. In this study, the effects of numerical solution parameters such as time step, grid size and convergence criteria, and grid boundary integration on the pressure distribution between rotor and bearing are investigated for radial air bearing with high values of clearances (to 250 $\mu \mathrm{m})$.

\footnotetext{
* Sorumlu Yazar/Corresponding author: abdurrahimdal@gazi.edu.tr / Tel: +90 3125823464
} 


\section{GIRIŞS (INTRODUCTION)}

Basınçlı hava yatakları (BHY) ile desteklenmiş yatak-rotor sistemlerinde, yatak ve rotor yüzeyleri arasındaki yağlayıcı hava filmi; yatak üzerine yerleştirilen hava besleme delikleri ile bu iki yüzey arasına iletilerek sağlanabilir (Şekil 1). Dolayısıyla bu yüzeyler arasındaki hava filmi ve havanın akışı BHY ile desteklenmiş rotorun dinamiğini doğrudan etkilemektedir. BHY ile desteklenmiş rotorun dinamik karakteristiklerinin analizi üzerine yapılan ilk araştırmalar da yatak ve rotor arasındaki akışın modellenmesi ve bu modelin çözümlenmesi üzerine olmuştur [1]. Havanın sıkıştırılabilirlik özelliğinden dolayı, akışkanın (havanın) hareketi doğrusal olmayan bir karakteristik sergilemektedir [2]. Yapılan teorik [3] ve deneysel [4] çalışmalar sonucunda, çeşitli kabullerden yola çıkılarak, yatak ve rotor arasındaki havanın hareketi Reynold's denklemi olarak adlandırılan parabolik bir diferansiyel denklem ile ifade edilmektedir [5]. Bu denklem, yatak ve rotor arasında kalan boşluk miktarına, akışkanın termodinamik özelliklerine, rotorun açısal hızına, zamana ve rotorun yatay ve dikey eksenlerdeki hareketine bağlı olarak değişen bir ifade olup, akışkanın hızına veya basıncına göre düzenlenebilir [6]. Reynold's denklemi sayısal metotlar kullanılarak çözülüp, yatak ve rotor arasındaki basınç dağılımı, rotora etkiyen basınç kuvvetleri ve dolayısıyla yatağın dinamik karakteristikleri (yük taşıma kapasitesi, yay katılığı, vb.) belirlenebilir [7]. BHY'lerde, basınç dağılımı, genellikle havanın hem sönüm hem de katılık etkisini içeren bir sonuç veren pertürbasyon metodu kullanılarak hesaplanmaktadır [8]. Bunun yanı sıra sonlu elemanlar [9], sonlu elemanlar tabanlı metotlar [10, 11] ve sonlu farklar metotları da kullanılmaktadır [12]. Ancak bu çözüm metotlarında bir grid noktasının hesaplanması için en az beş bilinmeyenli bir denklem takımının çözülmesi gerekmektedir. Dolayısıyla her bir grid noktasının basınç değerlerinin hesaplanması için işlemci zamanı ve işlemci yükü de paralel olarak artmaktadır [13]. Basınç dağılımının hesaplanması için kullanılan çözüm tekniklerinin iyileştirilmesi ve daha hızlı sonuçlar veren algoritmaların kullanılması son zamanlarda BHY konusundaki çalışmalarda sıklıkla yer almaktadır. Bunun yanı sıra [14]'de verilen momentum denklemi gibi doğrusal olmayan akış problemleri için sıklıkla kullanılan paket programları da (Fluent, Ansysis-CFX) Reynold's denklemi çözümlerinde tercih edilebilmektedir [15]. Czolczynski, [16] Reynold's denkleminin çözümünde değişen yönlü kapalı nümerik integrasyon metodunu kullanarak, $38 \mu \mathrm{m}$ boşluk miktarına sahip basınçlı hava yatağı için basınç dağılımını hesaplamış ve çözümü hızlandırmıştır. Piekos, [17] Reynold's denklemini pertürbasyon çözümüne göre daha hızlı bir çözüm yapan iki farklı algoritma kullanarak yatak ve rotor arasındaki basınç dağılımını hesaplamıştır. Wang ve ark., [18] yaptıkları çalışmada hibrid metot olarak adlandırdıkları, DTM (differential transform method) ve SOR (successive over relaxation) metotları kullanarak daha hızlı çözümler elde etmişlerdir. Lo ve ark., da [19] Newton metodu ve SOR metodu kullanarak yatak ve rotor arasındaki basınç dağılımlarını hesaplamışlar ve elde ettikleri sonuçları birbiri ile karşılaştırmışlardır. Fakat kullandıkları çözüm teknikleri küçük boşluk miktarları (50 $\mu \mathrm{m}$ den daha küçük) için daha iyi sonuçlar vermiştir. Chen ve ark. [20] $10 \mu \mathrm{m}$ ve $15 \mu \mathrm{m}$ radyal boşluğa sahip basıç̧l hava yataklarının çalışma şartlarının (besleme basıncı, hız vb.) yatak katılığına olan etkilerini teorik ve deneysel incelemişlerdir. Yaptıkları çalışmada SOR metodunu kullanarak yataklardaki basınç dağılımlarını elde etmişlerdir. Zhang ve ark. [21] mikro elektronik cihazların rotorlarının yataklanması için tasarladıkları basınçlı hava yataklarının performanslarını teorik olarak incelemişlerdir. Basınç dağılımlarının hesaplanması için literatürdeki metotlardan farklı olarak Spektral Toplama metodunu kullanmışlardır. $\mathrm{Bu}$ metot ile silindirik ve eksenel koordinatlardaki basınç değişimini Chebyshev ve trigonometrik seriler ile ifade etmişlerdir. $\mathrm{Bu}$ metot ile sonlu farklar ve sonlu elemanlar metotlarına göre daha doğru sonuçlar elde edildiğini söylemişlerdir. Li ve ark. [22] yaptıkları teorik çalışmada $20 \mu \mathrm{m}$ daha küçük radyal boşluk miktarına sahip basınçlı hava yataklarındaki, basınç dağılımının hesaplanması için yeni bir sonlu farklar metodu önermişlerdir. Önerdikleri metot ile orifislerdeki akışın geri beslemeli kontrolünü yaparak, basınç dağılımını sonlu farklar metodu ile ayrıklaştırdıkları Reynold's denklemini çözerek elde etmişler ve pertürbasyon, SOR ve sonlu farklar metodu ile yapılan çalışmalara göre çözümü hızlandırmışlardır.

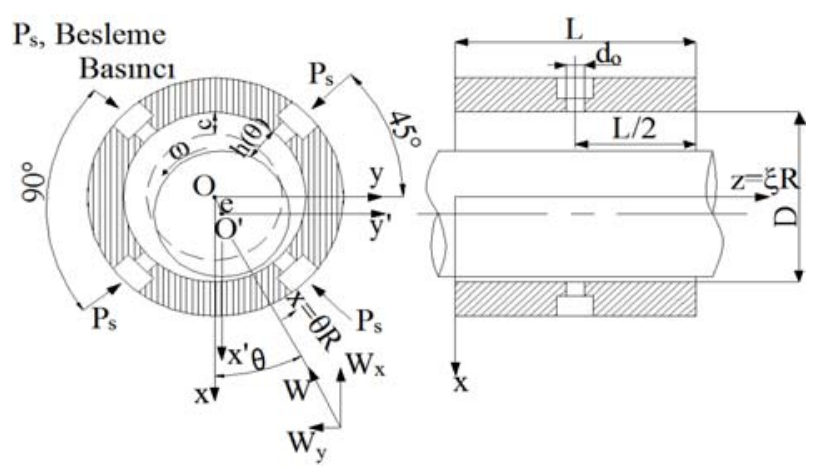

Şekil 1. Basınçlı hava yatağı

(Externally pressurized air lubricated bearing) [28]

BHY konusunda yapılan çalışmaların büyük bir çoğunluğu küçük boşluk miktarına sahip yataklar $(100 \mu \mathrm{m}$ den daha küçük) üzerinedir. BHY'lerin boşluk miktarları azaldıkça, imalat edilebilirliği de zorlaşmakta buna paralel olarak fiyatları artmaktadır. Ancak yatak ve rotor arasındaki boşluk miktarının artması ile birlikte basınç dağılımının hesaplanmasında ıraksama problemleri ortaya çıkmaktadır. Ayrıca nümerik çözüm için silindirik bir geometriye sahip yatağın iç yüzeyi herhangi bir noktasından açıldığ varsayılarak dikdörtgen bir grid oluşturulduğunda, yatağın açıldığı varsayılan noktalardaki (dikdörtgen gridin sınırlarındaki) basınç değerlerinin de hesaplanması gerekmektedir. $\mathrm{Bu}$ çalışmada daha büyük boşluk miktarlarına sahip (250 $\mu$ m'ye kadar) yataklar için, basınç 
dağılımının hesaplanması sırasında ortaya çıkan ıraksama problemleri ve birleşme çizgisi (dikdörtgen gridin sınırları) üzerindeki noktaların basınç değerlerinin hesaplanması için çözüm önerileri sunulmuştur. Bu kapsamda yatak ve rotor arasındaki havanın akışı Reynold's denklemi kullanılarak modellenmiş ve değişen yönlü nümerik integrasyon (DYKNI) metodu kullanılarak çözülmüştür. DYKNİ metodunun doğasında bulunan rraksama problemine zaman adımı ve yakınsama kriterinin değiştirilmesi ile çözüm aranmıştır.

\section{MATEMATÍKSEL MODEL (MATHEMATICAL MODEL)}

BHY'de (Şekil 1), besleme deliklerinden iletilen basınçlandırılmış hava, yatak ve rotor yüzeyleri arasında ince bir yağlayıcı film oluşturarak rotor dinamiğinin önemli parametreleri olan yay katılığını ve sönümü sağlar. BHY'lerde, yatak ve rotor arasındaki havanın hareketi; yatakların geometrilerine ve havanın termodinamik özelliklerine bağlı olarak akışkanın viskozitesinin sabit olduğu, Navier-Stokes denklemindeki atalet kuvvetinin ve cisim kuvvetlerinin çok küçük olduğu ve ince yağlayıcı film geometrisinden dolayı basıncın bu ince filmin kalınlığ boyunca değişmediği kabulleri altında modellenmektedir [4]. Bu kabuller ile birlikte, Navier-Stokes denklemleri ve süreklilik denklemi düzenlenerek yatak ve rotor arasındaki basınç dağılımının matematiksel ifadesi olan Reynold's denklemi, silindirik koordinatlarda ve boyutsuz formda, Eş. 1'deki gibi verilebilir [29].

$\frac{\partial}{\partial \theta}\left[\mathrm{PH}^{3} \frac{\partial \mathrm{P}}{\partial \theta}\right]+\frac{\partial}{\partial \xi}\left[\mathrm{PH}^{3} \frac{\partial \mathrm{P}}{\partial \xi}\right]+\Gamma_{\mathrm{s}} \mathrm{P}_{\mathrm{s}} \mathrm{H} \phi(\mathrm{P})=\Lambda \frac{\partial}{\mathrm{R} \partial \theta}(\mathrm{PH})+\sigma \frac{\partial}{\partial \mathrm{t}}(\mathrm{PH})$

Eş. 1'de geçen boyutsuz boşluk fonksiyonu, rotor eksantrikliğine $(\varepsilon)$ bağlı olarak $\mathrm{H}=(1+\varepsilon \cos (\theta))$ şeklinde ifade edilmektedir. Debi ifadesindeki besleme parametresi Eş. 2'de ve orifis fonksiyonu Eş. 3'de verilmiştir [30].

$$
\begin{aligned}
& \Gamma_{0}=\frac{12 \pi \mathrm{d}_{0}^{2} \mu \sqrt{\mathrm{R}^{0} \mathrm{~T}^{0}}}{\mathrm{p}_{\mathrm{a}} \mathrm{c}^{2} \Delta \theta \Delta \xi} \\
& \Phi(\mathrm{P})= \begin{cases}\sqrt{\frac{2 \mathrm{k}}{\mathrm{k}-1}\left[\left(\frac{\mathrm{p}_{\mathrm{d}}}{\mathrm{p}_{\mathrm{u}}}\right)^{\frac{2}{\mathrm{k}}}-\left(\frac{\mathrm{p}_{\mathrm{d}}}{\mathrm{p}_{\mathrm{u}}}\right)^{\frac{\mathrm{k}+1}{\mathrm{k}}}\right.} & ; 1>\frac{\mathrm{p}_{\mathrm{d}}}{\mathrm{p}_{\mathrm{u}}} \geq \mathrm{r}_{\mathrm{p}} \\
\frac{2 \mathrm{k} \frac{1}{2}}{\mathrm{k}+1}\left(\frac{2}{\mathrm{k}+1}\right)^{\frac{1}{\mathrm{k}-1}} & ; \frac{\mathrm{p}_{\mathrm{d}}}{\mathrm{p}_{\mathrm{u}}}<\mathrm{r}_{\mathrm{p}}\end{cases}
\end{aligned}
$$

\section{REYNOLD'S DENKLEMININ NÜMERIK ÇÖZÜMÜ \\ (NUMERICAL SOLUTION OF REYNOLD'S EQUATION)}

Bu çalışmada Reynold's denklemi direk nümerik çözüm tekniklerinden olan DYKNI metodu kullanılarak çözümlenmiştir.

\subsection{Grid Oluşturulması (Formed Grid)}

Nümerik çözüm için, Şekil 2‘de gösterildiği gibi, silindirik geometriye sahip yatağın iç yüzeyi, herhangi bir noktasından açıldı̆̆ varsayılarak dikdörtgen bir grid oluşturulabilir. Eş. 1'de verilen boyutsuz Reynold's denkleminin $\theta$ koordinatı yatak iç yüzeyinin çevresel koordinat eksenini ifade etmekte olup, 0 ile $2 \pi$ arasında, $\xi$ koordinatı ise boyutsuz yatak uzunluğunu ifade eden koordinat ekseni olup, 0 ile L arasında değerler alacaktır. Oluşturulan grid, koordinat adımları $\Delta \theta$ ve $\Delta \xi$ olacak şekilde $\mathrm{N}_{\mathrm{z}}=\mathrm{L} / \Delta \xi$ ve $\mathrm{N}_{\mathrm{x}}=\mathrm{L} / \Delta \theta$ sayıda parçalara bölünebilir. Dolayısıyla grid, sinırdaki noktalarla birlikte $\left(\mathrm{N}_{\mathrm{x}}+1\right) \times\left(\mathrm{N}_{\mathrm{z}}+1\right)$ sayıda noktadan oluşacaktır [27].

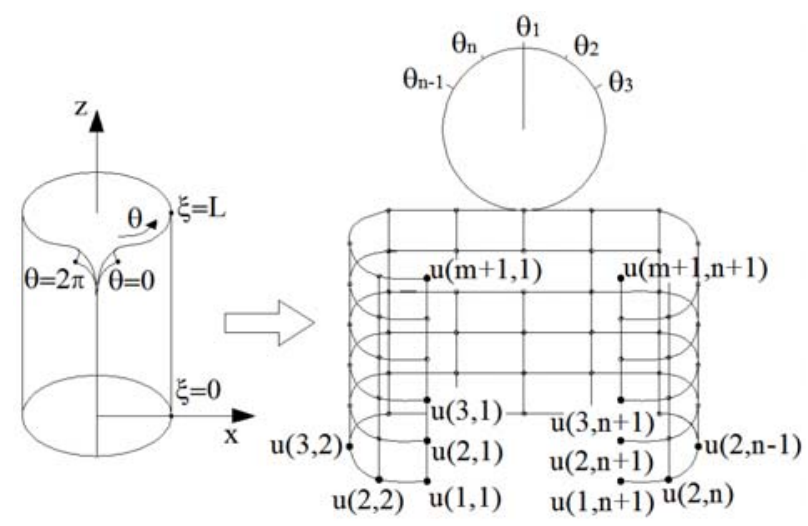

Şekil 2. Dikdörtgen gridin oluşturulması (Formed rectangular grid)

\subsection{Reynold's Denkleminin DYKNİ Metodu ile Çözümü (Aplication of Alternating Direction Implicit Method for Reynold's Equation)}

DYKNI metodu genel olarak birden fazla koordinatta değişen kısmi diferansiyel denklemlerinin çözümü için sonlu farklar metodunun genişletilmiş bir formudur [16]. Nümerik integrasyondan önce, Eş. 1'de verilen Reynold's denklemi, [27]'de detaylı şekilde açıklandığı gibi analitik olarak türevleri alınıp, Eş. 4'deki gibi yeniden düzenlenebilir.

$$
\begin{aligned}
& -3 \mathrm{H}^{2}\left(\frac{\partial \mathrm{H}}{\partial \theta} \frac{\partial \Theta}{\partial \theta}+\frac{\partial \mathrm{H}}{\partial \xi} \frac{\partial \Theta}{\partial \xi}\right)-\mathrm{H}^{3}\left(\frac{\partial^{2} \Theta}{\partial \theta^{2}}+\frac{\partial^{2} \Theta}{\partial \xi^{2}}\right)+\frac{\Lambda \mathrm{H}}{\mathrm{P}} \frac{\partial \Theta}{\partial \theta}+ \\
& 2 \Lambda \mathrm{P} \frac{\partial \mathrm{H}}{\partial \theta}+\frac{\sigma \mathrm{H}}{\mathrm{P}} \frac{\partial \Theta}{\partial \mathrm{t}}+2 \sigma \mathrm{P} \frac{\partial \mathrm{H}}{\partial \mathrm{t}}=2 \dot{\mathrm{M}}
\end{aligned}
$$

Şekil 3'de gösterildiği gibi, çözüm için tam bir zaman adımının iki eşit parçaya bölünmesi ile, ilk yarım zaman adımında bir eksen yönünde; ikinci yarım zaman adımında ise diğer eksen yönünde çözüm yapılabilir. DYKNİ metodu ile ilk yarım zaman adımında $u_{i-1, j}{ }^{n+1 / 2}, u_{i, j}{ }^{n+1 / 2}$ ve $u_{i+1, j}{ }^{n+1 / 2}$ noktaları çözülüp, ikinci yarım zaman adımında ise ilk yarım zamanda elde edilen çözüm kullanılarak, $\mathrm{u}_{\mathrm{i}, \mathrm{j}-1}{ }^{\mathrm{n}+1}$, $\mathrm{u}_{\mathrm{i}, \mathrm{j}}{ }^{\mathrm{n}+1}$ ve $\mathrm{u}_{\mathrm{i}, \mathrm{j}+1}{ }^{\mathrm{n}+1}$ hesaplanabilir. Eş. 4'de verilen ifadenin DYKNİ şeması [24]'de detaylı şekilde açıklanmıştır. 


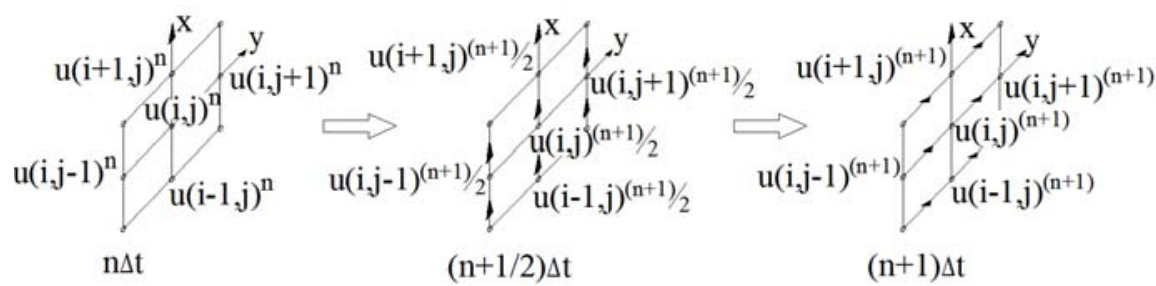

Şekil 3. DYKNİ şeması (ADI scheme) [24]

\subsection{Başlangıç Şartları, Sınır Şartları ve Nümerik Çözümün Yakınsama Kriteri \\ (Initial Condition, Boundary Condition and Convergence Criteria)}

İki ucu atmosfere açık olan BHY'nin nümerik çözümü için, başlangıçta yatağın içerisindeki basınç, atmosfer basıncında tanımlanmış ve aşağıdaki sınır şartları kullanılmıştır.

(a) Yatağın her iki ucu atmosfere açıktır, $\mathrm{P}(0, \theta)=\mathrm{P}(\mathrm{L}, \theta)=\mathrm{P}_{\mathrm{a}}$ (b) Birleşme çizgisi üzerindeki basınçlar birbirine eşittir, $\mathrm{P}(\xi, 0)=\mathrm{P}(\xi, 2 \pi)$ (c) Basınç dağılımı yatak merkezine göre simetriktir, $\mathrm{P}(0 \rightarrow \mathrm{L} / 2, \theta)=\mathrm{P}(\mathrm{L} / 2 \rightarrow \mathrm{L}, \theta)$ (d) Basınç dağılımı periyodiktir, $\mathrm{P}(\xi, \theta)=\mathrm{P}(\xi, \theta+2 \pi)$ ve $(\partial \mathrm{P} / \partial \theta)_{\theta}=(\partial \mathrm{P} / \partial \theta)_{\theta+2 \pi}$

Basınç değerlerinin bir önceki zaman adımında hesaplanan değerler ile karşılaştıran bir yakınsama kriteri, Eş. 5'deki gibi tanımlanmıştır [29].

$$
\operatorname{maks}\left\{\left|P_{i, j}{ }^{n+1}-P_{i, j}{ }^{n}\right| / P_{i, j}{ }^{n+1}\right\} \leq Y_{k}
$$

\subsection{Silindirik Geometrinin Düzlemsel Dikdörtgen Grid ile Ifade Edilmesi Problemi (Boundary Condition for Cylindirical Grid)}

Basınç dağılımının hesaplanması için yatak, silindirik geometrisi boyunca açılarak düzlemsel bir ızgara (grid) yapı oluşturulmuştur (Şekil 2). Dolayısıyla birleşme çizgisi olarak tanımlanan bu hat boyunca basınç değerleri aynı olmak zorundadir. Yani $\mathrm{u}(1,1)=\mathrm{u}(1, \mathrm{n}+1)$, $\mathrm{u}(2,1)=\mathrm{u}(2, \mathrm{n}+1), \ldots, \mathrm{u}(\mathrm{m}+1,1)=\mathrm{u}(\mathrm{m}+1, \mathrm{n}+1)$ şeklindedir. $B \mathrm{u}$ grid yapının DYKNİ metodu ile çözümünde üst ve alt kenarlarındaki basınç değerlerinin sınır şartı olarak verilmesine karşın, sağ ve sol kenarlarının basınç değerleri sadece başlangıç şartı olarak verilebilmektedir. Bunun yanı sıra birleşme çizgisi üzerindeki basınç değerleri rotorun eksantrikliğine bağlı olarak değişmektedir ve rotor simülasyonunun her adımı için tekrar hesaplanması gerekmektedir. Bu çalışmada birleşme çizgisindeki basınç değerlerinin iteratif olarak hesaplanması için, üçüncü mertebeden ileri ve geri farklar metotları ve silindirik yatak geometrisinden dolayı oluşan periyodik sınır şartı (Bölüm 3.4) kullanılarak elde edilen bir yöntem önerilmiştir. Bu yöntemde birleşme çizgisi üzerindeki basınç değerlerinin hesaplanması için birleşme çizgisi ve gerisindeki basınç değerleri kullanılarak Eş. 6'da verildiği gibi üçüncü mertebeden ileri farklar ve ilerisindeki basınç değerleri kullanılarak Eş. 7'de verildiği gibi üçüncü mertebeden geri farklar metotları ile açılmıştır [25]. $\left.\frac{\partial \mathrm{P}}{\partial \theta}\right|_{\theta}=\frac{-2 \mathrm{P}_{\mathrm{i}, 2}+3 \mathrm{P}_{\mathrm{i}, \mathrm{N}_{\mathrm{x}}+1}-6 \mathrm{P}_{\mathrm{i}, \mathrm{N}_{\mathrm{x}}}+\mathrm{P}_{\mathrm{i}, \mathrm{N}_{\mathrm{x}}-2}}{6 \Delta \theta}$

$\left.\frac{\partial \mathrm{P}}{\partial \theta}\right|_{\theta+2 \pi}=\frac{-\mathrm{P}_{\mathrm{i}, 3}+6 \mathrm{P}_{\mathrm{i}, 2}-3 \mathrm{P}_{\mathrm{i}, 1}-2 \mathrm{P}_{\mathrm{i}, \mathrm{N}_{\mathrm{x}}}}{6 \Delta \theta}$

Diğer taraftan periyodik sınır şartında verildiği gibi birleşme çizgisi üzerindeki basınç değerleri birbirine eşit olacaktır. Dolayısıyla Eş. 6 ve Eş. 7 birbirine eşitlenip düzenlenerek birleşme çizgisi üzerindeki basınç değerlerinin hesaplanması için $\left(\mathrm{P}_{\mathrm{i}, 1}=\mathrm{P}_{\mathrm{i}, \mathrm{N}}\right)$ Eş. 8'de verilen ifade önerilebilir.

$\mathrm{P}_{\mathrm{i}, 1}=\frac{-2 \mathrm{P}_{\mathrm{i}, 3}+8 \mathrm{P}_{\mathrm{i}, 2}}{6}$

\section{SONUÇLAR VE TARTIŞMALAR (RESULTS AND DISCUSSIONS)}

\subsection{Basınç Dağıllımları (Pressure Distribution)}

Basınç dağılımı için DYKNİ metodu ile elde edilen çözüm şeması, Matlab yazılımı ile kodlanmış ve farklı parametreler için benzetimler gerçekleştirilmiştir. Basınç dağılımının hesaplanması için hazırlanan koda ait akış şeması Şekil 4'de verilmiştir. Matlab ortamında hazırlanan bu kod, basınçlı hava yatağının geometrik parametrelerinin ve havanın termodinamik özelliklerinin giriş olarak verilmesi ile başlatılmakta ve yatağın geometrik özellikleri ile birlikte çözüm gridi oluşturulmaktadır. Daha sonra bu grid üzerindeki başlangıç basınç dağılımı ve sınır şartları tanımlanmaktadır. DYKNİ metodu teorisine uygun olarak (Bölüm 3.2), ilk yarım zaman adımında, çözüm gridinin bir yönündeki düğüm noktaları için katsayılar matrisi oluşturulup, basınç değerleri hesaplanmaktadır. Bunun ardından, bu basınç değerleri ikinci yarım zaman adımı için ilk şart olarak tanımlanmakta ve çözüm gridinin diğer yönündeki düğüm noktaları için katsayılar matrisi oluşturulup, basınç değerleri hesaplanmaktadır. Son olarak, ikinci yarım zamanında elde edilen basınç değerleri yakınsama kriteri ile kontrol ettirilmektedir. Basınç değerleri için hesaplanan bağıl hata yakınsama kriterinden büyük olduğu sürece, bu basınç değerleri başlangıç basıncı olarak tanımlanarak, algoritma tekrar katsayılar matrisi oluşturulmak üzere başa dönmekte ve bağıl hata yakınsama kriterinden küçük olana kadar çözüm devam etmektedir. 
Çözüm için $0,05 \mathrm{~m}$ boyunda, yarı çapı $0,025 \mathrm{~m}$ olan ve çıkış çapı $0,003 \mathrm{~m}$ olan 4 orifisli tipik bir BHY kullanılmıştır. Özellikleri verilen BHY'nin yatak ve rotor arasındaki basınç değerlerinin yatak çevresi ve yatak boyunca dağılımı, 2 atm besleme basıncı için, üç boyutlu olarak Şekil 5'de verilmiştir. Rotor ve yatak eş merkezli olduğu için basınç dağılımı, sınır şartlarına bağlı olarak beklendiği gibi tam simetrik oluşmaktadır. Ayrıca sürekli bir hava beslemesi olduğu için beklendiği gibi en yüksek basınç orifislerin karşısında oluşmakta ve yatağın uçlarının atmosfere açık olmasından dolayı; yatağa 2 atm basınçta giren hava, her iki uçtan atmosfer basıncına düşerek çıkmaktadır. Şekil 4'de verilen basınç dağılımı niteliksel olarak literatürdeki [16], [19] ve [26]. çalışmaları ile uyumluluk göstermektedir

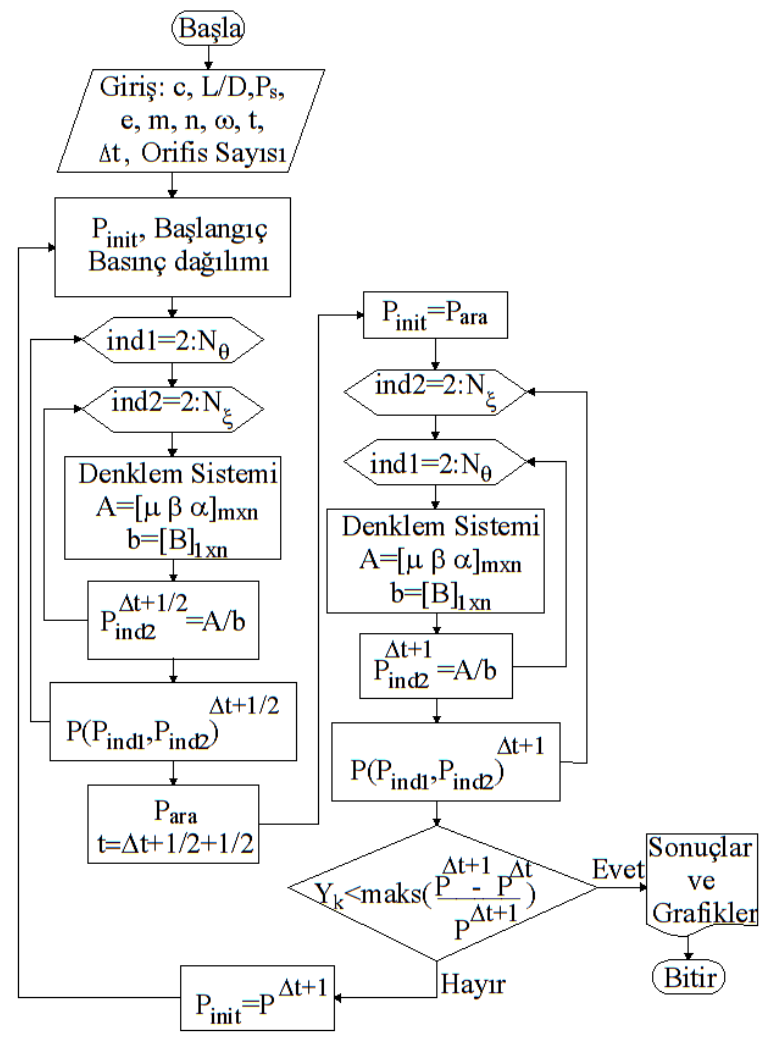

Şekil 4. Basınç dağılımının hesaplanması için akış şeması (Flowchart for calculation of pressure distribution)

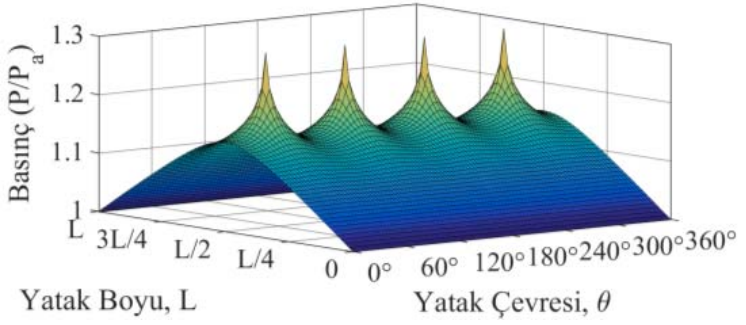

Şekil 5. Basınç dağılımı ( $\varepsilon=0, \mathrm{c}=250 \mu \mathrm{m})$ (Pressure distribution)

Yatak ile rotor eş merkezli değilse, yatak ve rotor arasındaki boşluğa bağlı olarak basınç dağılımı da değişecektir. Şekil 6'da yatak içerisindeki basınç oluşumu farklı eksantriklik (c) ve boşluk miktarları (c) için verilmiştir. Görüldüğü gibi radyal boşluk miktarının az olduğu yerde beklendiği gibi basınç daha yüksek; fazla olduğu yerlerde ise basınç daha düşük çıkmaktadır.

\subsection{Grid Boyutlarının Basınç Dağılımına Etkisi (Effect of Grid Size on Pressure Distribution)}

Düzlemsel dikdörtgen gridi oluşturan nokta sayısı ne kadar fazlaysa yani grid noktaları arasındaki mesafe ne kadar küçükse, basınç değerlerinin çözünürlüğü artacaktır. Ancak doğal olarak çözüm için geçen süre, nokta sayısı ile artmaktadır. Grid boyutlarının çözüme olan etkisinin incelenmesi için Reynold's denklemi Tablol'de verilen farklı grid boyutlarında çözülerek sonuçlar birbirleriyle karşılaştırılmıştır.

Tablo 1. Grid Boyutları (Grid Size)

\begin{tabular}{llllll}
\hline & 1. Grid & 2. Grid & 3. Grid & 4. Grid & 5. Grid \\
\hline $\mathrm{N}_{\xi} \times N_{\theta}$ & $16 \times 24$ & $32 \times 48$ & $48 \times 72$ & $64 \times 96$ & $80 \times 120$ \\
$\Delta \theta$ & 0,2500 & 0,1250 & 0,0883 & 0,0625 & 0,0500 \\
$\Delta \xi$ & 0,2618 & 0,1309 & 0,0873 & 0,0654 & 0,0524 \\
\hline
\end{tabular}

Tablo 2'de farklı gridler için hesaplanan basınç değerleri, yatak içerisindeki basınç dağılımı yatak çevresine göre periyodik ve yatak merkezine göre simetrik olduğu için yalnızca yatak boyunun yarısı için verilmiştir. Görüldüğü gibi basıncın yüksek olduğu yerlerde grid sayısının artması ile göreceli hata azalmaktadır. (Şekil 7). Bu analiz basıncın en yüksek olduğu orifis çıkış noktası için yapılacak olursa göreceli hata $1 ., 2$. ve 3 . gridler için $\% 1$ civarındadır. 3 . ve 4. gridler arasında \% 0,7 değişim olurken 4 . ve 5 . gridler arasındaki değişim \% 0,5 'lerin de altına düşmektedir. Buradan yola çıkılarak bu çalışma için Tablo 2'deki bilgisayar CPU süreleri de göz önüne alınarak 4. gridin yeterli olduğu kabul edilmiştir.

Tablo 2. Farklı grid boyutları için basınç değerleri (Pressure value for different grid size)

\begin{tabular}{llllll}
\hline \multicolumn{6}{l}{ Basınç Değerleri $\mathrm{P} / \mathrm{P}_{\mathrm{a}}$} \\
\hline$\xi(\theta=\pi / 4)$ & 1. Grid & 2. Grid & 3. Grid & 4. Grid & 5. Grid \\
0 & 1 & 1 & 1 & 1 & 1 \\
0,0125 & 1,0996 & 1,0957 & 1,0946 & 1,0937 & 1,0923 \\
0,025 & 1,2512 & 1,2751 & 1,2894 & 1,2992 & 1,3061 \\
CPU Time & 55,55 & 112,56 & 185,24 & 250,85 & 341,17 \\
\hline
\end{tabular}

\subsection{Zaman Adımının Basınç Dă̆ılımına Etkisi}

(Effect of Time Step on Pressure Distribution)

DYKNI metodunun yapısal olarak doğal bir kararlılık (stabilite) problemi bulunmaktadır ve BHY çözümleri için zaman adımlarının en fazla 0,001 s olması önerilmektedir [16]. Fakat literatürde bulunan BHY'nin nümerik çözümlerinde, genellikle $10^{-7} \mathrm{~s}$ zaman adımı kullanılmaktadır [17]. Bu çalışmada daha yüksek boşluk miktarları kullanıldığ için $(\mathrm{c}>100 \mu \mathrm{m}) 10^{-7}$ s'de çözüm elde edilememiş ve Reynold's denklemi daha küçük zaman adımları kullanılarak çözülmüştür. 


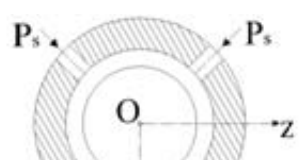

$P_{s} \cdot \frac{1}{2} \cdot P_{s}$

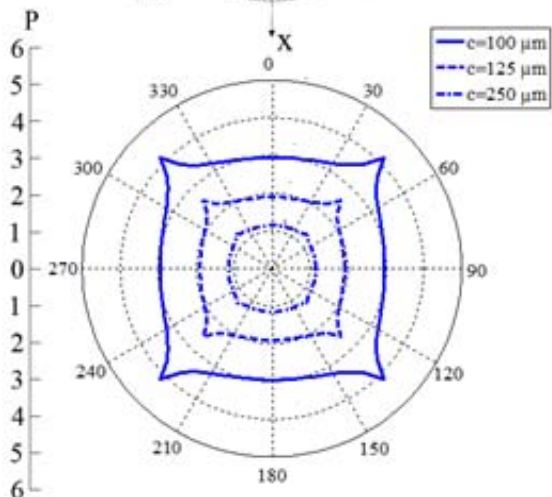

a.

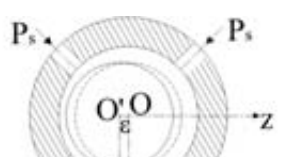

$P_{s} \cdot P_{s}$

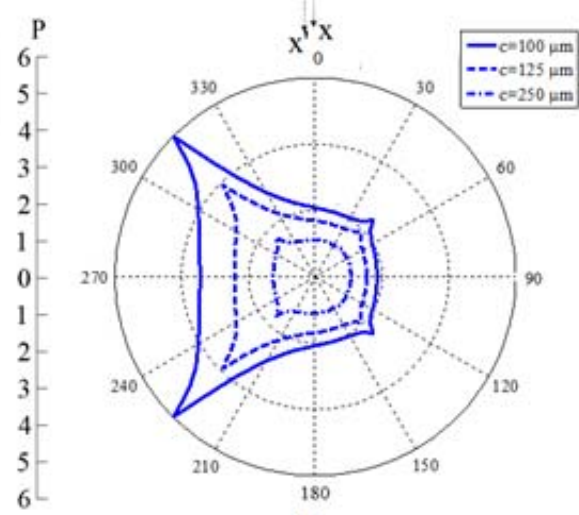

b.

Şekil 6. Farklı radyal boşluk miktarları için basınç dağılımları a. $\varepsilon=0$, b. $\varepsilon=0,5$ (Pressure distribution at circumferential direction for different radial clearances)

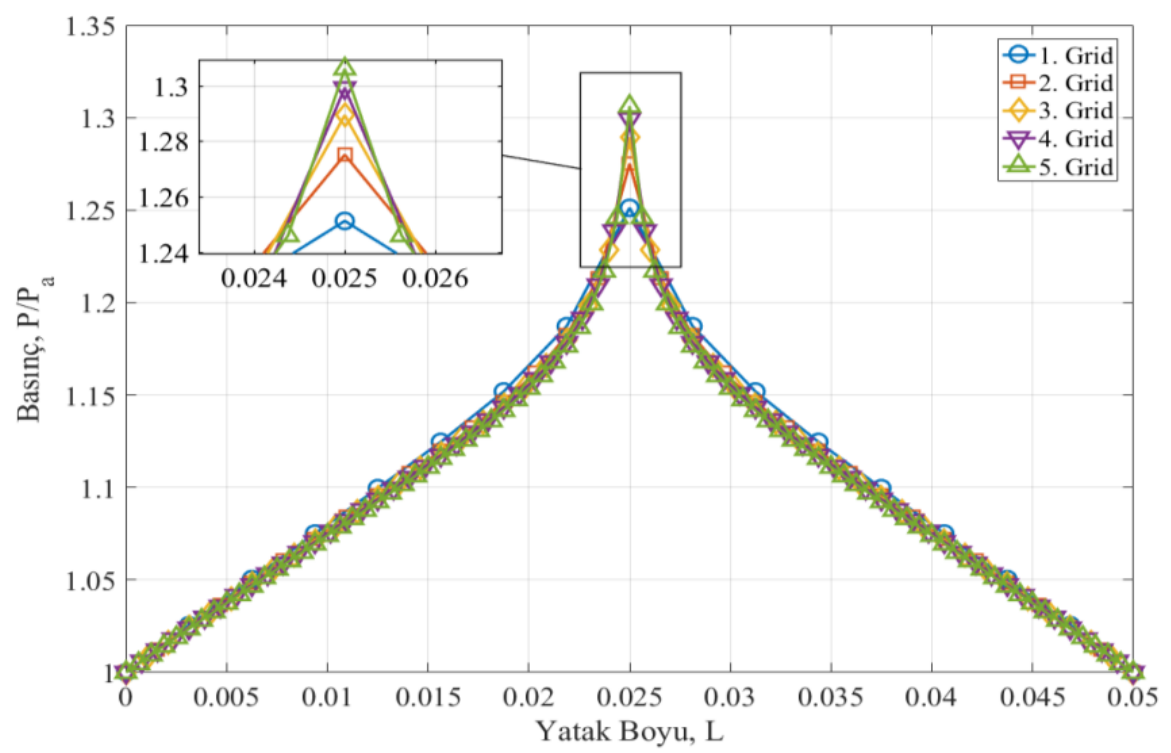

Şekil 7. Farklı grid boyutları için basınç değerlerinin yatak boyuna göre değişimi (Pressure values vs bearing length for different grid size)

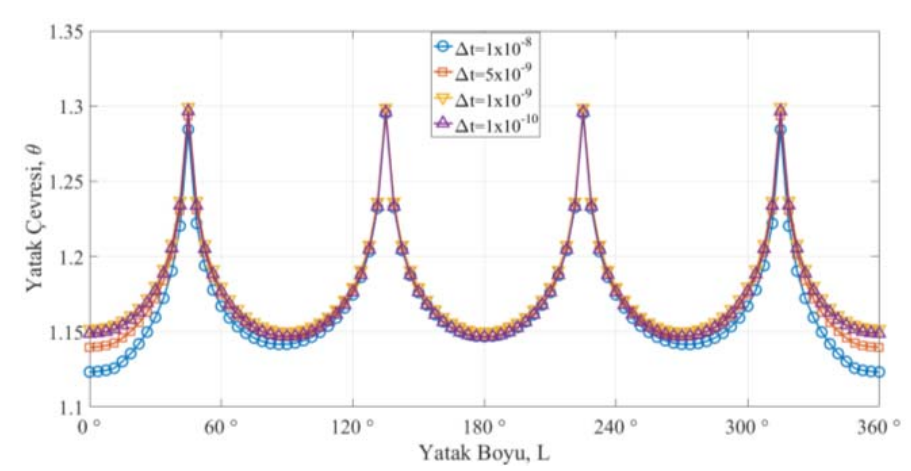

Şekil 8. Farklı zaman adımları için basınç değerlerinin yatak çevresine göre değişimi (Pressure distribution vs circumference direction for different time step) 
Dal ve Karaçay / Journal of the Faculty of Engineering and Architecture of Gazi University 32:1 (2017) 159-169

Tablo 3. Farklı zaman adımları için basınç değerleri (Pressure values for different time step)

\begin{tabular}{|c|c|c|c|c|c|}
\hline \multicolumn{6}{|c|}{ Zaman Adımları $(\Delta \mathrm{t})$ - Basınç Değerleri $(\mathrm{P} / \mathrm{Pa})$} \\
\hline$\theta(\xi=\mathrm{L} / 2)$ & $1 \times 10^{-7} \mathrm{~s}$ & $1 \times 10^{-8} \mathrm{~s}$ & $5 \times 10^{-9} \mathrm{~s}$ & $1 \times 10^{-9} \mathrm{~s}$ & $5 \times 10^{-10} \mathrm{~s}$ \\
\hline 0 & - & 1,0928 & 1,1105 & 1,1301 & 1,1292 \\
\hline$\pi / 4$ & - & 1,2283 & 1,2393 & 1,2510 & 1,2497 \\
\hline$\pi / 2$ & - & 1,1305 & 1,1382 & 1,1456 & 1,1434 \\
\hline CPU Time & - & 37 & 68,50 & 250,85 & 426,20 \\
\hline
\end{tabular}

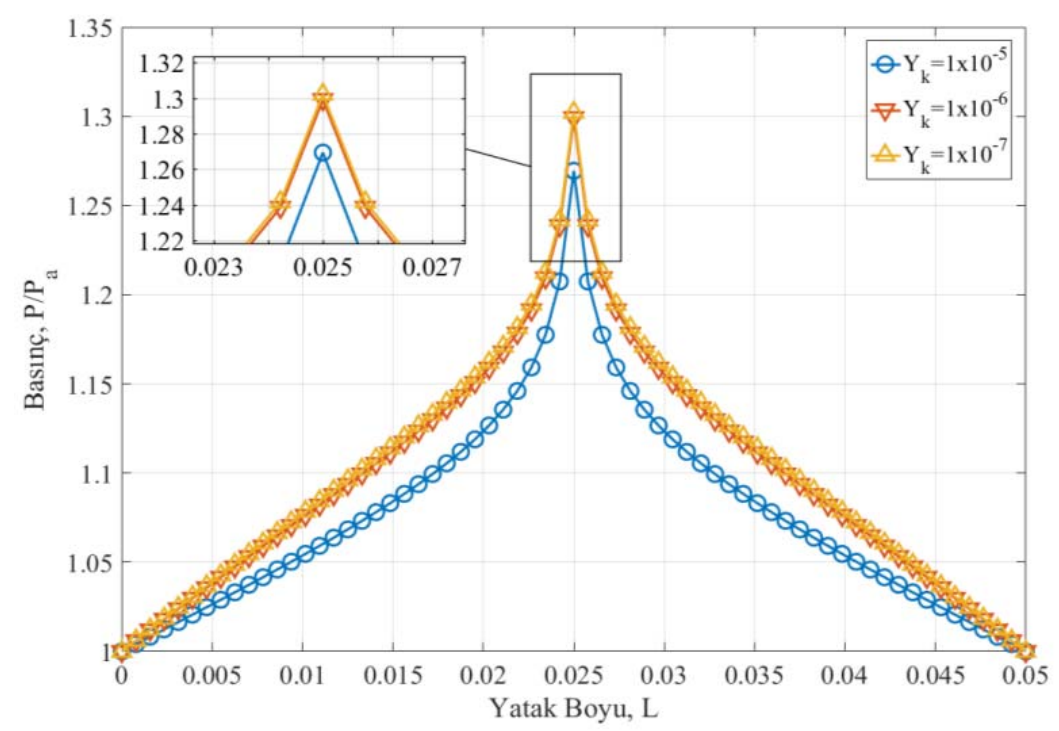

Şekil 9. Farklı yakınsama kriterleri için basınç değerlerinin yatak boyuna göre değişimi (Pressure distribution vs bearing length for different convergence criteria)

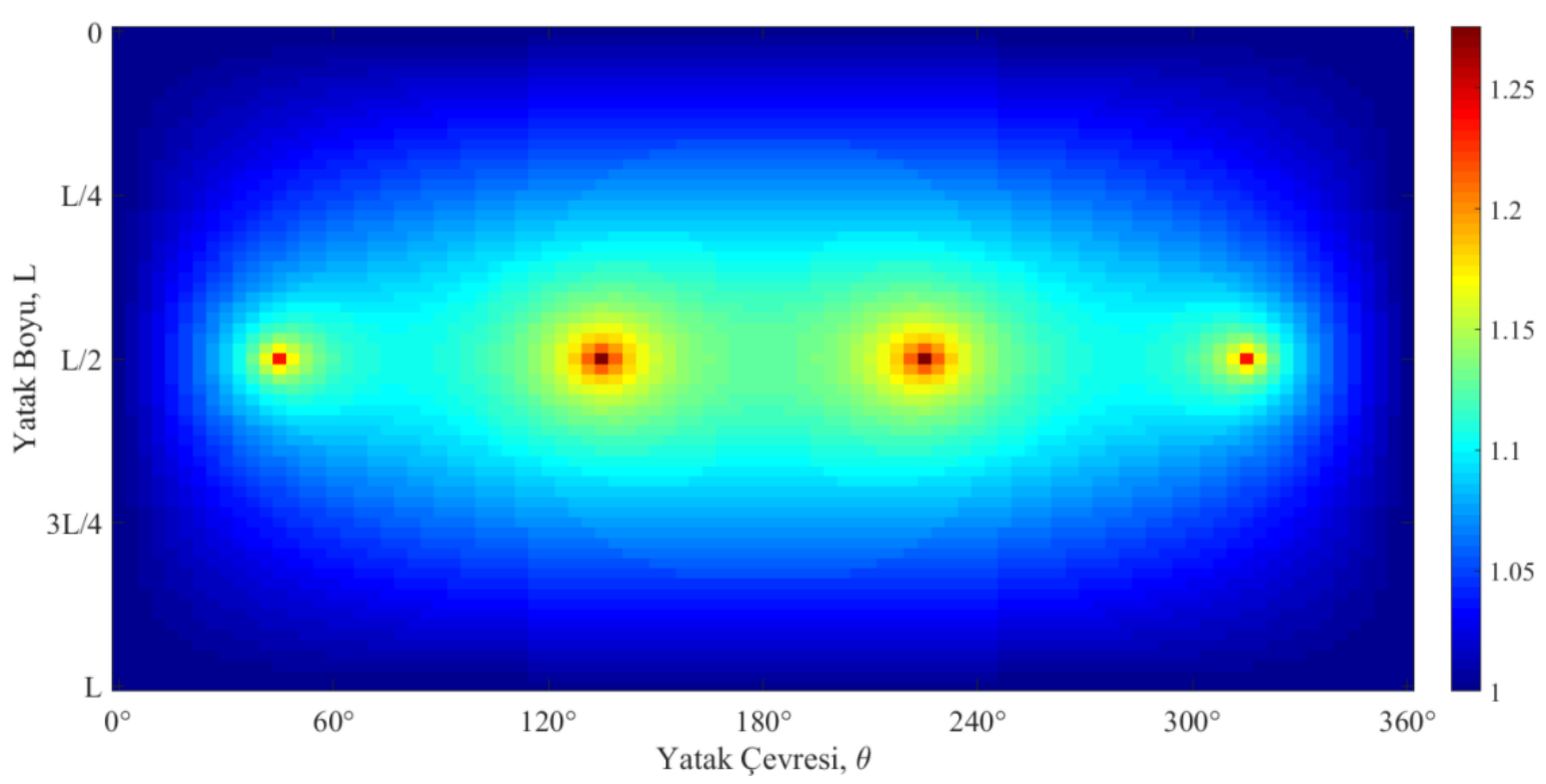

Şekil 10. Yöntem kullanılmadan elde edilen basınç dă̆glımı (Pressure distribution without adopt method) 


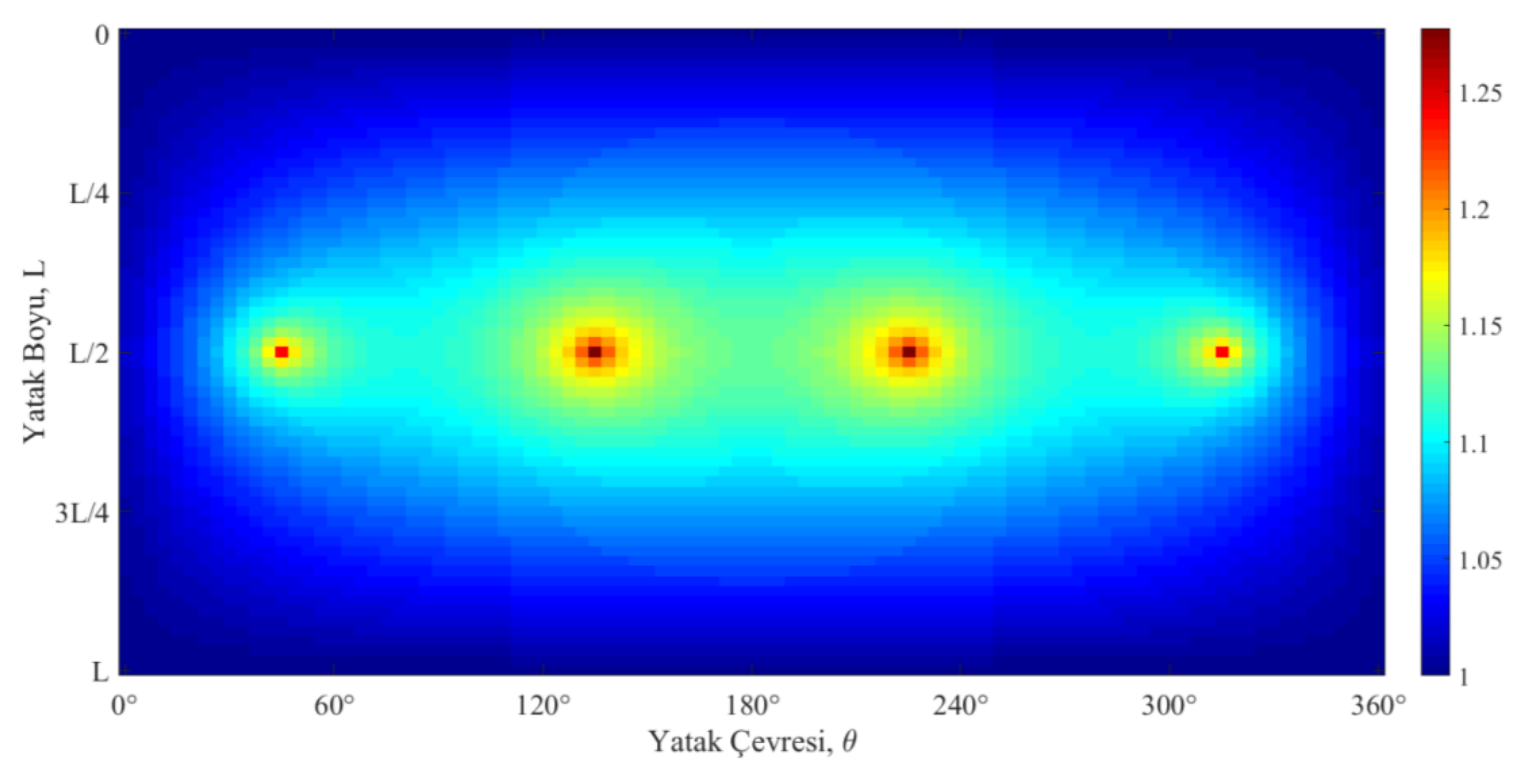

Şekil 11. Birleşme çizgisi için yöntem kullanılması ile elde edilen basınç dağılımı (Pressure distribution obtained with adopt method for only grid boundary integration)

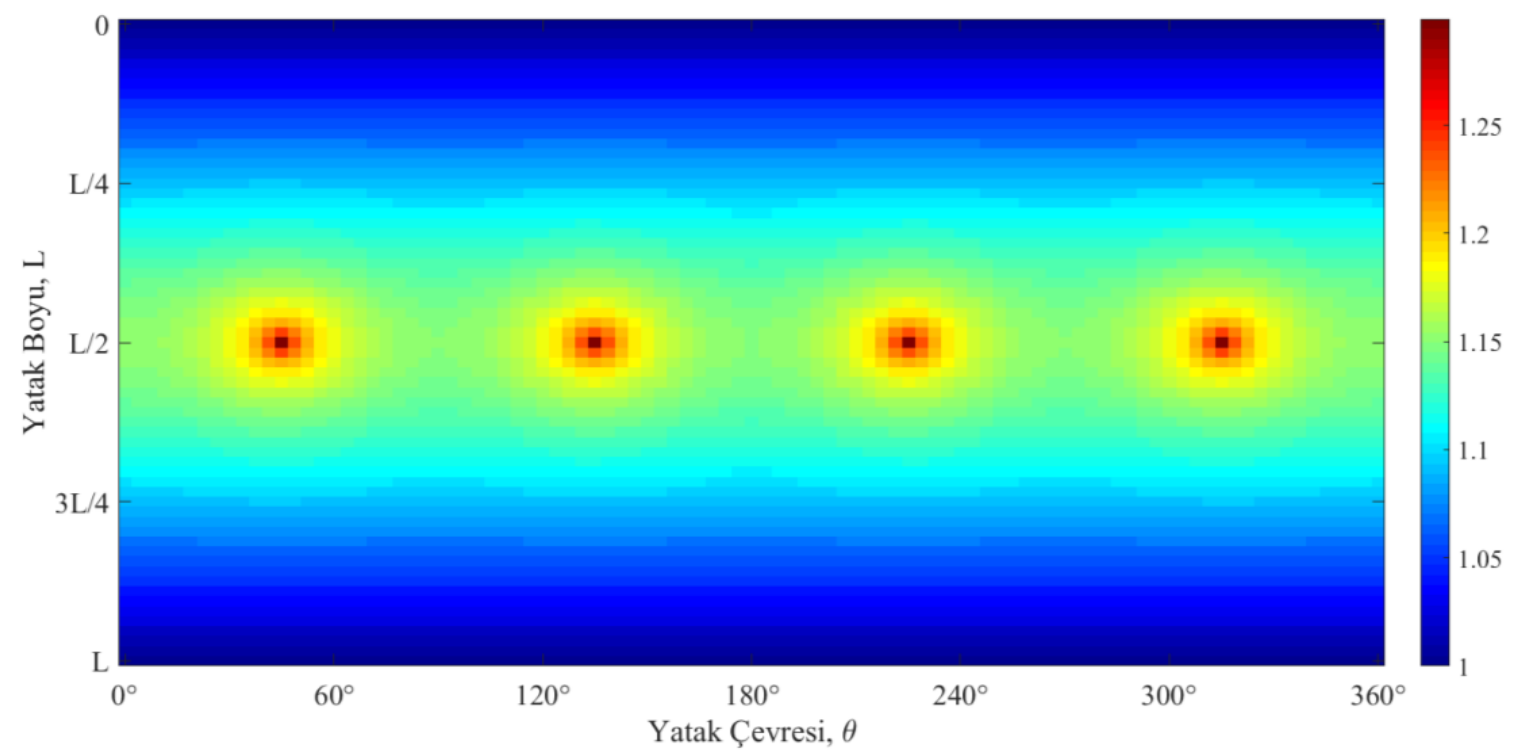

Şekil 12. Birleşme çizgisi ve birleşme çizgisinin ilerisi ve gerisi için yöntemin kullanılması ile elde edilen basınç dağılımı (Pressure distrubition obtained with adopt method at boundary grid integration and forward and back of boundary grid integration)

\subsection{Yakınsama Kriterinin Basınç Dă̆ılımına Etkisi (Effect of Convergence Criteria on Pressure Distribution)}

Çözümün yakınsamasının değerlendirilebilmesi için, basınç değerlerini, bir önceki zaman adımında hesaplanan değerler ile karşılaştıran bir yakınsama kriteri tanımlanmıştır (Eş. 5). Yakınsama kriterinin basınç dağılımına etkisi Şekil 9'da gösterilmiştir. Tablo 4'de farklı yakınsama kriterleri için yatak boyundaki basınç değerleri verilmiştir.

Görüldüğü gibi yakınsama kriteri çözümün yakınsamasını, yani akışın kararlılığını etkilediği için göreceli hata yatak başı ve yatak ortası arasında artmaktadır (Şekil 9). Göreceli hata $10^{-5}$ ve $10^{-6}$ arasında $\% 2$ civarında iken $10^{-6}$ ve $10^{-7}$ 166 arasında \% 0,2'nin altına düşmektedir. Yakınsama kriteri, göreceli hata değerleri ile beraber bu çalışma için Tablo 4'deki bilgisayar CPU süreleri de göz önüne alınarak $10^{-6}$ değerinin yeterli olduğu kabul edilmiştir.

Tablo 4. Farklı yakınsama kriterleri için basınç değerleri (Pressure values for different convergence criteria)

\begin{tabular}{lllll}
\hline $\begin{array}{l}\text { Yakinsama } \\
\text { Kriterleri }\left(\mathrm{Y}_{\mathrm{k}}\right)\end{array}$ & $\xi=0$ & $\xi=0,0125$ & $\xi=0,025$ & CPU Time \\
\hline $10^{-5}$ & 1 & 1,0654 & 1,2694 & 97,18 \\
$10^{-6}$ & 1 & 1,0937 & 1,2992 & 250,85 \\
$10^{-7}$ & 1 & 1,0961 & 1,3022 & 420,33 \\
\hline
\end{tabular}




\subsection{Silindirik Gridin Sinır Şartı Problemi}

(Boundary Condition Problem of Cylindirical Grid)

Yatağın silindirik geometrisinin, çözümde kullanılmak üzere açık bir dikdörtgen gride dönüştürülmesi sebebiyle ortaya çıkan açık kenarlardaki basınç değerlerinin elde edilmesi için Eş. 8'de verilen ifade kullanılmış ve elde edilen basınç dağılımları Şekil 10-12'de gösterilmiştir. Önerilen yöntem kullanılmadan verilen sınır şartları ile birlikte Reynold's denklemi DYKNİ metodu kullanılarak çözdürülmüş ve elde edilen basıç̧ değerlerinin yatak boyu ve yatak çevresine göre dağılımları Şekil 10'da verilmiştir. Ancak çözümde kullanılan silindirik grid yapının birleşme çizgisi olarak tanımlanan sınırları basınç dağılımını etkilemekte ve yatak çevresinde periyodik (sürekli) bir dağ 11 ım elde edilememektedir.

Fiziksel olarak beklenildiği gibi yatak ve rotor arasındaki basınç dağılımının yatak çevresinde periyodik ve simetrik olabilmesi için bu birleşme çizgisi üzerindeki basınç değerlerinin hesaplanması gerekmektedir. Şekil 11'de gösterilen basınç dağılımında birleşme çizgisi üzerindeki noktaların basınç değerleri Eş. 8'de önerilen çözüm yöntemi kullanılarak hesaplanmıştır. Ancak birleşme çizgisi üzerindeki basınç değerleri hesaplanmasına rağmen henüz yatak çevresine göre periyodik ve simetrik bir basınç dağılımı elde edilememiştir.

Grid noktaları arasındaki mesafe küçük olduğundan birleşme çizgisi üzerindeki basınç değerlerinin hesaplanması için birleşme çizgisinin sağındaki ve solundaki çizgilerde de (2. ve n. çizgi) basınç değerleri Eş. 8 'de verilen ifade kullanılarak hesaplanabilir. Şekil 12'de birleşme çizgisinin bir sağındaki ve bir solundaki çizgi üzerindeki basınç değerlerinin önerilen çözüm yöntemi kullanılarak hesaplanması ile elde edilen basınç dağılımı gösterilmiştir. Fiziksel olarak beklendiği gibi yatak ve rotor arasındaki basınç dağılımı hem yatak boyunun merkezine göre hem de yatak çevresinin merkezine göre periyodik ve simetrik elde edilmiştir.

\section{SONUÇLAR (CONCLUSIONS)}

$\mathrm{Bu}$ çalışmada büyük boşluk miktarlarına sahip basınçlı hava yataklarında, yatak ve rotor arasındaki basınç dağılımının nümerik çözümünde kullanılan DYKNİ metodunun raksama problemine çözüm sunulmuştur. Elde edilen sonuçlara göre $250 \mu \mathrm{m}$ boşluk miktarına sahip basınçlı hava yatağında yatak ve rotor arasındaki basınç dağılımını ifade eden Reynold's denkleminin nümerik çözümü için zaman adımı $10^{-9} \mathrm{~s}$ ve yakınsama kriteri $10^{-6}$ ve $64 \times 96$ noddan oluşan ve adım aralıkları $0,0625 \times 0,0654$ olan grid optimum parametreler olarak elde edilmiştir.

Yapılan bu çalışma ile elde edilen sonuçlar [10], [22] ve [31] çalışmalarda yer alan üç boyutlu basınç dağılımı grafikleri ile karşılaştırıldığında, her ne kadar basınçlı hava yataklarının geometrik özellikleri ve çalışma parametreleri farklı da olsa, birleşme çizgisi üzerindeki basınç

değerlerinin, orifislerdeki maksimum basıncın ve orifislerden yatak kenarlarına dağılan basıncın formsal olarak uygunluk gösterdiği görülmüştür. Bu çalışma ile elde edilen diğer bir sonuç ise; Reynold's denkleminin nümerik çözümünde kullanılan dikdörtgen gridin birleşme çizgisi üzerindeki noktaların basınç değerlerinin yüksek mertebeden geri ve ileri farklar kullanılarak elde edilebileceğidir. Bunun yanı sıra fiziksel olarak beklenen (silindirik yatak geometrisine bağlı olarak hem yatak boyuna hem de yatak çevresine göre simetrik) basinç dağılımının elde edilmesi için önerilen bu yöntemin birleşme çizgisi üzerindeki basınç değerlerinin hesaplanmasının yanı sıra birleşme çizgisinin bir adım sağındaki ve bir adım solundaki çizgi üzerinde bulunan noktaların basınç değerleri için de kullanılması gerekmektedir.

\section{SIMMGELER (SYMBOLS)}

$\mathrm{c}$

$\mathrm{d}_{0}$

D

$\mathrm{h}$

$\mathrm{H}$

k

L

$\dot{\mathrm{M}}$

$\mathrm{n}$

$\mathrm{N}_{\mathrm{x}}, \mathrm{N}_{\mathrm{z}}, \mathrm{N}_{\theta}, \mathrm{N}_{\xi}$

$\mathrm{O}$

$\mathrm{O}^{\prime}$

$\mathrm{p}_{\mathrm{a}}$

$p_{d}$

$\mathrm{p}_{\mathrm{s}}$

$\mathrm{p}_{\mathrm{u}}$

P

$r_{p}$

$\mathrm{R}$

$\mathrm{R}^{0}$

$\mathrm{t}$

$\Delta \mathrm{t}$

$\mathrm{T}^{0}$

$\mathrm{u}_{\mathrm{i}, \mathrm{j}}$

$\mathrm{x}, \mathrm{y}, \mathrm{z}$

$\mathrm{Y}_{\mathrm{k}}$

$\varepsilon$

$\theta, \xi$

$\theta_{\mathrm{i}} \mathrm{i}=1,2, . ., \mathrm{n}$

$\mu$

$\Delta \xi, \Delta \theta$

$\sigma$

$\Phi(\mathrm{P})$

$\Gamma_{0}$

$\Lambda$
Yatak ve rotor arasındaki boşluk, $\mathrm{m}$

Besleme deliği çapı, m

Yatak çapı, m

Yatak ve rotor merkezleri arasındaki mesafe, $\mathrm{m}$

Hava filmi kalınlığ $1, \mathrm{~m}$

Boyutsuz hava filmi (h/c)

Is1 kapasitesi oran1

Yatak boyu, $\mathrm{m}$

Boyutsuz kütle debisi

Zaman adımı sayıs

Koordinatlar boyunca grid nokta say1s1

Yatak merkezi

Rotor merkezi

Atmosfer basinc1, atm

Besleme deliği çıkıș basıncı, atm

Besleme basinc1, atm

Besleme deliği giriş basıncı, atm

Boyutsuz basınç

Kritik basınç oranı

Yatak yarıçapı, m

Gaz sabiti, $\mathrm{J} / \mathrm{kgK}^{\circ}$

Zaman, s

Zaman adımı, s

Mutlak sicaklık, $\mathrm{K}^{\circ}$

Kismi diferansiyel fonksiyon

Kartezyen koordinatlar

Yakınsama kriteri

Ekzantriklik oran1 (e/c)

Boyutsuz silindirik koordinat eksenleri

Yatak çevresi, rad

Dinamik vizkozite, Pas

Boyutsuz koordinatlarda grid adımları

Sıkıştırılabilirlik sayısı

Besleme deliği fonksiyonu,

Boyutsuz besleme parametresi

Yatak say1s1

$\mathrm{P}^{2}$ 


\section{TEŞEKKÜR (ACKNOWNLEDGEMENT)}

Bu çalışma TÜBITAK ARDEB-1001 kapsamında 112M847 numaralı proje ile desteklenmiştir. Bu çalışmaya olanak sağlayan TÜBİTAK'a teşekkür ederiz.

\section{KAYNAKLAR (REFERENCES)}

1. Fuller D.D., A review of research in the field of gaslubricated bearings, NASA Report, 2429, USA, 5-50, 1970.

2. Uneeb M., Theoretical Investigation of Whirl Instability In Externally Pressurized Gas Journal Bearings, PhD Thesis, Imperial College of Science, Techgnologyand Medicine, London, UK, 29-92, 1992.

3. Ausman J.S., The fluid dynamic theory of gas lubricated bearing, Trans. Amer. Soc. Lub., Engrs., 79 (6), 12181224, 1957.

4. Gross W.A., Investigation of whirl in externally pressurized air-lubricated journal bearings, Trans. Amer. Soc. Mech. Engr. J. Basic Eng., 84, 132-140, 1962.

5. Heinrich G., The theory of the externally pressurized bearings, 1st International Symposium on GasLubricated Bearing, USA, 42, 1959.

6. Wang J., Design of Gas Bearing Systems for Precision Applications, PhD. Thesis, Technische Universiteit, Eindhoven, 1-35, 1993.

7. Lund J.W., A theoretical analysis of whirl instability and pneumatic hammer for a rigid rotor in pressurized gas journal bearing, An International Journal on the Science and Technology of Friction, Lubrication and Wear, 12 (2), 154-166, 1967.

8. Xu C., Jiang S., Dynamic Analysis of a Motorized Spindle With Externally Pressurized Air Bearings, Journal of Vibration and Acoustics, 137 (4), 041001, 2015.

9. Du J., Zhang G., Liu T., To S., Improvement on load performance of externally pressurized gas journal bearings by openning pressure-equalizing grooves, Tribology International, 73 (Mayıs 2014), 156-166, 2014.

10. Charki A., Diop K., Champmartin S., Ambari A. Numerical simulation and experimental study of thrust air bearings with multiple orifices, International Journal of Mechanical Sciences, 72, 28-38, 2013.

11. Gao S, Cheng K., Chen S., Ding H., Fu H., Computational design and analysis of aerostatic journal bearing with application to ultra-high speed spindles, Proc. of the Inst. of Mech. Eng., Part C: Journal of Mechanical Engineering Science, 095440621663934, 2016.

12. Wang X., Xu Q., Wang B., Zhang L., Yang H., Peng Z., Effects of surface waviness on the static performance of aerostatic journal bearing, Tribology International, 103, 394-405, 2016.

13. Wang C., Yau H., Theoretical analysis of high speed spindle air bearings by a hybrid numerical method,
Applied Mathematics and Computation, 217 (5), 20842096, 2010.

14. Bilgili M., Sivrioğlu M., Numerical Analysis of Pem Fuel Cell at Different Mea Thicknesses and Operating Pressure Conditions, Journal of the Faculty of Engineering Architecture of Gazi University, 31 (1), 51-63, 2016.

15. Chang S.H., Chan, C.W., Jeng Y.R., Numerical analysis of discharge coefficients in aerostatic bearings with orifice-type restrictors, Tribology International, 90 (October 2015), 157-163, 2015.

16. Czolczynski K., Ling F.F., Winer W.O., Rotordynamics of Gas-Lubricated Journal Bearing Systems, Springer, NewYork, 1-48, 1999.

17. Piekos E.S., Numerical Simulation of Gas- Lubricated Journal Bearings For Microfabricated Machines, PhD. Thesis, Department of Aeronautics and Astronautics Massachusetts Institute of Technology, Boston, 15$108,2000$.

18. Wang C., Application of a hybrid method to the nonlinear dynamic analysis of a flexible rotor supported by a spherical gas-lubricated bearing system, Nonlinear Analysis:theory, Method \& Applications, 70 (5), 2035-2053, 2009.

19. Lo Y., Wang C.C., Lee Y.H., Performance analysis of high-speed spindle aerostatic bearings, Tribology International, 38 (1), 5-14, 2005.

20. Chen Y.S., Chiu C.C., Cheng Y.D., Influence of operational conditions and geometric parameters on stifnness of aerostatic journal bearings, Precision Engineering, 34 (4), 722-734, 2010.

21. Zhang W.M., Zhou J.B., Meng G., Performance and stability analysis of gas lubricated journal bearings in MEMS, Tribology International, 44 (7), 887-897, 2011.

22. Li Y., Zhou K., Zhang Z., A flow difference feedback iteration method and its application to high-speed aerostatic journal bearings, Tribology International, 84 (Apr 2015), 132-141, 2015.

23. Haentjens T., Hout K., Alternating direction implicit finite difference schemes for the Heston-Hull-White partial differential equation, The Journal of Computational Finance, 16 (1), 83-110, 2012.

24. Dal A., Basınçlı hava yatağıyla desteklenmiş yatakrotor sisteminin dinamik karakteristiklerinin belirlenmesi, Yüksek Lisans Tezi, Gazi Üniversitesi, Fen Bilimleri Enstitüsü, Ankara, 126-128, 2014.

25. Chapra S.C., Canale, P.R., Numerical Methods for Engineers, McGraw-Hill, 3th Edition, 832-848, 1988.

26. Viktorov V., Belforte G., Raparelli T., Modeling and identification of gas journal bearings: externally pressurized gas bearing results, Journal of Tribology, 127 (3), 548-556, 2005.

27. Karaçay T., Basınçlı Hava Yataklı Rotor Dinamiğinin Teorik ve Deneysel İncelenmesi, Sonuç Raporu, ARDEB-1001-112M847, TUBİTAK, 2015.

28. Dal A., Karaçay T., On the Numerical and Experimental Analysis of Internal Pressure in Air Bearings, International Journal of Mechanical, Aerospace, Industrial, Mechatronic and Manufacturing Engineering, 9 (7), 1232-1237, 2015. 
29. Dal A., Karaçay T., On Dynamics of an Externally Pressurized Air Bearing with High Values of Clearance: Effect of Mass Flow Rate, Proceedings of the World Congress on Engineering, Vol-II, LondonU.K., 3-6 July, 2014.

30. Dal A., Karaçay T., Vibration of Two Degrees of Freedom Air Bearing rotor System With Asymmetric
Supply Pressure, International Conference on Engineering Vibration, Ljubljana-Slovenia, 519-528, 710 September, 2015.

31. Otsu Y., Somaya K., Yoshimoto S., High-speed stability of a rigid rotor supported by aerostatic journal bearings with compound restrictors, Tribology International, 44 (1), 9-17, 2011. 
